\title{
WIF-1 gene inhibition and Wnt signal transduction pathway activation in NSCLC tumorigenesis
}

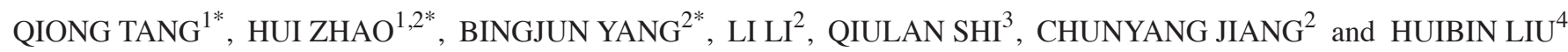 \\ Departments of ${ }^{1}$ Respiratory Medicine and ${ }^{2}$ Thoracic Surgery, Tianjin Union Medical Center, Tianjin 300121; \\ ${ }^{3}$ School of Foreign Languages, North China University of Science and Technology, Tangshan, Hebei 063001; \\ ${ }^{4}$ Office of Clinical Drug Trial Institution, The Affiliated Tumor Hospital of Xinjiang Medical University, \\ Urumqi, Xinjiang 830011, P.R. China
}

Received June 28, 2015; Accepted November 1, 2016

DOI: $10.3892 / 01.2017 .5566$

\begin{abstract}
The aim of the present study is to explore the differential expression of key molecules associated with Wnt signaling in both clinical non-small cell lung cancer (NSCLC) tissue and adjacent normal lung tissue, and to discuss the tumorigenic role of the activation of Wnt signaling pathways in NSCLC. A total of 52 NSCLC patients were employed in the present study. Lung cancer tissue samples and paracarcinoma tissue samples were obtained from these patients, who had undergone surgical resection of their primary cancer. The cases were diagnosed by hematoxylin and eosin staining. Using reverse transcription-quantitative polymerase chain reaction and immunohistochemical straining, the messenger RNA (mRNA) and protein expression levels of Wnt inhibitory factor-1 (WIF-1) and important molecules associated with Wnt signaling pathways were detected. Compared with normal tissues, a marked decreased in the mRNA and protein expression levels of WIF-1, and an increase in $\beta$-catenin and cyclin D1 expression, were observed in tumor tissues. This suggests that the activation of the $\mathrm{Wnt} / \beta$-catenin signaling pathway may be closely associated with lymph nodal metastasis and lower pathological classification. However, no obvious difference could be observed in adenomatous polyposis coli (APC) expression levels between lung cancer tissues and adjacent tissues to the carcinoma. The activation of the Wnt/ $\beta$-catenin signaling pathway in NSCLC could be initiated by WIF-1
\end{abstract}

Correspondence to: Dr Huibin Liu, Office of Clinical Drug Trial Institution, The Affiliated Tumor Hospital of Xinjiang Medical University, 789 Suzhou East Road, Urumqi, Xinjiang 830011, P.R. China

E-mail: 1hbrose2010@126.com

Dr Chunyang Jiang, Department of Thoracic Surgery, Tianjin Union Medical Center, 190 Jieyuan Road, Hongqiao, Tianjin 300121, P.R. China

E-mail: chunyangjiang@126.com

*Contributed equally

Key words: WIF-1, Wnt, NSCLC, regulation, tumorigenesis gene inhibition without APC expression changes, and this may be different to the mechanism in other tumors.

\section{Introduction}

Wnt signaling pathways are important in controlling embryonic development, and are highly conserved in evolution (1). The members of these pathways are highly homologous between different species, from lower organisms such as Drosophila to mammals (1). Previous studies demonstrated that the processes of cell proliferation, organ formation and cell determination involve Wnt signal transduction $(1,2)$. In mature individuals, abnormal activation of Wnt signaling is closely associated with the occurrence of various diseases such as cancer $(1,2)$. It is generally accepted that, as the most important factor of Wnt signaling pathways in normal mature cells, $\beta$-catenin only acts as a type of intracellular cytoskeleton protein close to the cellular membrane, and forms a complex with E-cadherin to maintain homotypic cell adhesion by preventing cell movement (3). $\beta$-catenin is not usually present in the cytoplasm. Only under certain conditions, $\beta$-catenin accumulates in the cytoplasm, and it is then transferred to the nucleus (3). The activation of the $\mathrm{Wnt} / \beta$-catenin signaling pathway is considered to have effects on cell proliferation (3).

Previous studies revealed that the mechanism of abnormal activity of Wnt signaling in lung cancer is different from that in colorectal cancer $(4,5)$. One of the most significant differences is the activation of Wnt signaling pathways. Mutations and anomalous protein expression of $\beta$-catenin and adenomatous polyposis coli (APC), a tumor-suppressor protein that is a component of the $\mathrm{Wnt} / \beta$-catenin signaling pathway, are rarely observed in lung cancer $(4,5)$. This suggests that a specific mechanism of Wnt signaling pathways activation exists in lung tumors.

Multiple factors have been identified to serve a role in the regulation of Wnt signaling pathways (1). Wnt inhibitory factor-1 (WIF-1) is an important negative regulatory factor for this pathway, which belongs to the secreted frizzled-related proteins family (6). As the inhibitory factor upstream of the Wnt signaling pathways, WIF-1 is a highly conserved gene, and can inhibit the activation of these pathways by directly combining with Wnt signaling proteins (6). It is well known 
that WIF-1 could act as a tumor suppressor, and WIF-1 has been observed to be epigenetically silenced in various cancers (6).

Previous studies have shown that disordered methylation patterns in the regulation of gene expression, such as high methylation of tumor-suppressor genes, will lead to tumorigenesis (7). Previous studies reported that WIF-1 expression was significantly downregulated or even silenced by high methylation in its promoter region in non-small cell lung cancer (NSCLC) cell lines (8). Therefore, WIF-1 may be a key antagonist for Wnt signaling pathways to prevent the occurrence of lung cancer (9).

The present study aimed to explore the differential expression of key molecules associated with Wnt signaling pathways between clinical NSCLC and paracarcinoma tissue samples. In addition, the present study discusses the role of the activation of molecules associated with Wnt signaling pathways in the tumorigenic mechanism of NSCLC.

\section{Materials and methods}

Reagents. The RNAprep Pure kit (For Tissue) was purchased from Tiangen Biotech Co., Ltd. (Beijing, China). The RevertAid First Strand cDNA Synthesis kit was purchased from Fermentas (Thermo Fisher Scientific, Inc., Pittsburgh, PA, USA). A reverse transcription-quantitative polymerase chain reaction (RT-qPCR) kit (DRR01AM) was purchased from Takara Bio, Inc. (Otsu, Japan). Antibodies specific to $\beta$-catenin (catalog no., sc-7199) and $\beta$-actin (catalog no., sc-130300) were obtained from Santa Cruz Biotechnology, Inc. (Dallas, TX, USA). Anti-WIF-1 (catalog no., 5652) and anti-cyclin D1 (catalog no., 1044S) antibodies were purchased from Cell Signaling Technology, Inc. (Danvers, MA, USA). Peroxidase-conjugated ImmunoPure ${ }^{\circledR}$ goat anti-rabbit immunoglobulin $\mathrm{G}(\operatorname{IgG})$ [heavy $(\mathrm{H})+$ light $(\mathrm{L})]$ and biotinylated ImmunoPure ${ }^{\circledR}$ goat anti-mouse $\operatorname{IgG}(\mathrm{H}+\mathrm{L})$ (catalog no., MII0401) were purchased from Pierce (Thermo Fisher Scientific, Inc., Waltham, MA, USA). All other reagents used were analytical-grade laboratory chemicals from standard commercial suppliers.

Clinical cases selection and tissue samples treatment. Human NSCLC tissue samples and paracarcinoma lung tissue samples (used as control samples) were obtained from 52 patients [23 squamous carcinomas and 29 adenocarcinomas; tumor-node-metastasis (TNM) I, 24 cases and TNM II/III, 28 cases] from January 2012 to October 2013. These patients had undergone surgical resection of their primary cancer at the Department of Thoracic Surgery in Tianjin Union Medicine Centre (Hongqiao, China). Lung tissues were obtained by video-assisted thoracoscopic surgery to resect the pulmonary lobe containing the tumor, and then flash frozen in liquid nitrogen and stored at $-150^{\circ} \mathrm{C}$ or fixed in paraformaldehyde. All patients provided informed consent prior to sample collection, according to the institutional guidelines of Tianjin Union Medicine Centre. The present protocol was approved by the ethics committee of Tianjin Union Medicine Centre. The histological type and grade of tumor were classified on the basis of the World Health Organization criteria (10). The stage of each cancer was identified according to the International
Association for the Study of Lung Cancer and the American Thoracic Society criteria (11). All primary tumor tissues and control samples were diagnosed by hematoxylin and eosin (H\&E) staining. The frozen samples were used for RT-qPCR detection, while the tissues fixed in paraformaldehyde were used for immunohistochemical examination. The concentration of RNA was determined by the absorbance (A) 260/A280 ratio using a spectrophotometer (Bio-Rad Laboratories, Inc., Hercules, CA, USA).

Assessment of lung histopathology. Histopathological determination was performed as described previously (12). The lung tumor tissues were removed from the $10 \%$ formaldehyde storage solution, and then subjected to regular dehydration, paraffin embedding, sectioning and dewaxing. Upon H\&E staining, samples were subjected again to dehydration and sealing. Next, the tumor's histopathology was observed under a light microscope, and images were obtained.

RT-qPCR analysis. Total cellular RNA of lung tissues was isolated using the RNAprep Pure kit (For Tissue) (Tiangen Biotech Co., Ltd.) according to the manufacturer's protocol. Total RNA $(2.0 \mu \mathrm{g})$ was reverse transcribed into complementary DNA using the RevertAid First Strand cDNA Synthesis kit with oligo(dT). All primers were designed and synthesized by Takara Bio, Inc. The primer sequences, annealing temperatures and expected product sizes are listed in Table I. All PCR procedures were conducted in the MJ Research PTC-200 DNA Engine Thermal Cycler (Bio-Rad Laboratories, Inc.) using the following cycling parameters: $2 \mathrm{~min}$ of initial denaturation at $94^{\circ} \mathrm{C}$, followed by $30 \mathrm{sec}$ of denaturation at $94^{\circ} \mathrm{C}$, and 35 cycles of $30 \mathrm{sec}$ at $94^{\circ} \mathrm{C}$ (denaturing), $30 \mathrm{sec}$ at $58^{\circ} \mathrm{C}$ (annealing) and $30 \mathrm{sec}$ at $72^{\circ} \mathrm{C}$ (elongation), with a final extension at $72^{\circ} \mathrm{C}$ for $5 \mathrm{~min}$. The presence of PCR products was confirmed by gel electrophoresis on a $2 \%$ agarose gel and staining with ethidium bromide. Bands were visualized in a Gel Doc 1000 system (Bio-Rad Laboratories, Inc.). $\beta$-actin was used as an internal control in parallel for each replicate. Gel-Pro Analyzer software version 3.0 (Media Cybernetics, Inc., Rockville, MD, USA) was used to quantify the denisty of each band. The experiments were performed three times independently, and the mean value was used for comparison.

Immunohistochemical staining analysis. Immunohistochemical staining was used to detect the protein expression of WIF-1, $\beta$-catenin and cyclinD1, as described previously (13). Paraffin-embedded tissues were sectioned serially into 4- $\mu \mathrm{m}$-thick sections, which were then immersed in $10 \mathrm{mmol} / 1$ citrate buffer ( $\mathrm{pH}$ 6.0) and heated in a microwave oven at $100^{\circ} \mathrm{C}$ for $10 \mathrm{~min}$. Endogenous peroxidase activity was quenched with $3 \%$ hydrogen peroxide in methanol for $10 \mathrm{~min}$. Subsequently, the sections were blocked with $5 \%(\mathrm{v} / \mathrm{v})$ bovine normal serum (Tiangen Biotech Co., Ltd.) in PBS for $20 \mathrm{~min}$. Next, sections were incubated for $12 \mathrm{~h}$ or overnight at $4^{\circ} \mathrm{C}$ in a humidified chamber with the following primary antibodies: Rat polyclonal anti-human $\beta$-catenin (1:50; Santa Cruz Biotechnology, Inc.), rat polyclonal anti-human $\beta$-actin (1:50; Santa Cruz Biotechnology, Inc.), rat polyclonal anti-human WIF-1 (1:50; Cell Signal Technology, Inc.), rat polyclonal anti-human APC (1:50; Cell Signal Technology, Inc.) and rat polyclonal anti-human 
Table I. List of primers used for reverse transcription-quantitative polymerase chain reaction analyses.

\begin{tabular}{|c|c|c|c|}
\hline Gene name & Primer sequence $\left(5^{\prime}-3^{\prime}\right)^{\mathrm{a}}$ & Annealing temperature, ${ }^{\circ} \mathrm{C}$ & Product length, bp \\
\hline WIF-1 & $\begin{array}{l}\text { F: ATCCTGCACCTGCGACTACAG } \\
\text { R: GGCGACTTCTCGAAGTAGACC }\end{array}$ & 58.0 & 432 \\
\hline APC & $\begin{array}{l}\text { F: CGGAACATGCATGACTGATAC } \\
\text { R: GTCACGAGGTACGACCTCAGAT }\end{array}$ & 58.0 & 310 \\
\hline$\beta$-catenin & $\begin{array}{l}\text { F: AAGTTCTTGGCTATTACGACA } \\
\text { R: ACAGCACCTTCAGCACTCT }\end{array}$ & 58.2 & 375 \\
\hline Cyclin D1 & $\begin{array}{l}\text { F: CAGAAGTGCGAAGCTTAGGTCT } \\
\text { R: GTAGCAGGAGTAGTCCAGCGG }\end{array}$ & 58.0 & 420 \\
\hline$\beta$-actin & $\begin{array}{l}\text { F: CGTTGACATCCGTAACGACTCC } \\
\text { R: ATAGAGCCACCATTCCGACACAG }\end{array}$ & 56.0 & 660 \\
\hline
\end{tabular}

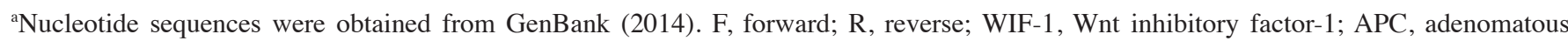
polyposis coli.

Table II. Clinicopathological factors of patients with adenocarcinoma or squamous carcinoma.

\begin{tabular}{lcc}
\hline Characteristics & $\begin{array}{c}\text { Patients with adenocarcinoma } \\
(\mathrm{n}=29), \mathrm{n}(\%)\end{array}$ & $\begin{array}{c}\text { Patients with squamous carcinoma } \\
(\mathrm{n}=23), \mathrm{n}(\%)\end{array}$ \\
\hline Gender & $18(62.07)$ & $14(60.87)$ \\
Male & $11(37.93)$ & $9(39.13)$ \\
Female & & $16(69.57)$ \\
Lymph nodal metastasis & $23(79.31)$ & $7(30.43)$ \\
Positive & $6(20.69)$ & $7(30.43)$ \\
Negative & & $10(43.48)$ \\
Pathological classification & $13(44.83)$ & $6(26.09)$ \\
High & $7(24.14)$ & 0.420 \\
Middle & $9(31.03)$ & $12(52.17)$ \\
Low & & $11(47.83)$ \\
Clinical stage & $12(41.38)$ & 0.438 \\
I & $17(58.62)$ & \\
II/III & & \\
\hline
\end{tabular}

cyclin D1 (1:50; Cell Signal Technology, Inc.). Thereafter, sections were washed three times with PBS, incubated with an appropriate biotinylated secondary antibody (1:1,000; Thermo Fisher Scientific, Inc.), washed three times and incubated at $4^{\circ} \mathrm{C}$ for $20 \mathrm{~min}$ with streptavidin-peroxidase (catalog no., CJ31H; Thermo Fisher Scientific, Inc.). Staining was visualized by adding 3,3'-diaminobenzidine (DAB Substrate; Roche Diagnostics GmbH, Mannheim, Germany), and then counterstained using hematoxylin. Sections were next rinsed in tap water, dehydrated through $70-100 \%$ graded alcohol, cleared in xylene and finally mounted in permanent mounting medium. Representative micrographs of the immunohistochemical results were acquired with a microscope camera system (FSX100; Olympus Corporation, Tokyo, Japan). Three sections were analyzed for each sample.

Statistical analysis. Data were presented as percentage or as means \pm standard deviation. Student's $t$-test and analysis of variance were used to detect differences in the mean values of the variables. Fisher's exact test or $\chi^{2}$ test was used as appropriately to analyze the differences in each variable. $\mathrm{P}<0.05$ was considered to indicate a statistically significant difference. All statistical analyses were performed with the software SPSS 13.0 (SPSS, Inc., Chicago, IL, USA).

\section{Results}

Clinicopathological characteristics of cases. A total of 29 cases with adenocarcinoma and 23 cases with squamous carcinoma were randomly selected and employed in the present study. The mean age of the patients in the adenocarcinoma group and in the squamous carcinoma group was 56.3 years (range, 38-73 years) and 53.8 years (range, 35-72 years), respectively. There were no differences in gender, nodal metastasis, pathological classification or clinical stage between the adenocarcinoma group and the squamous carcinoma group 


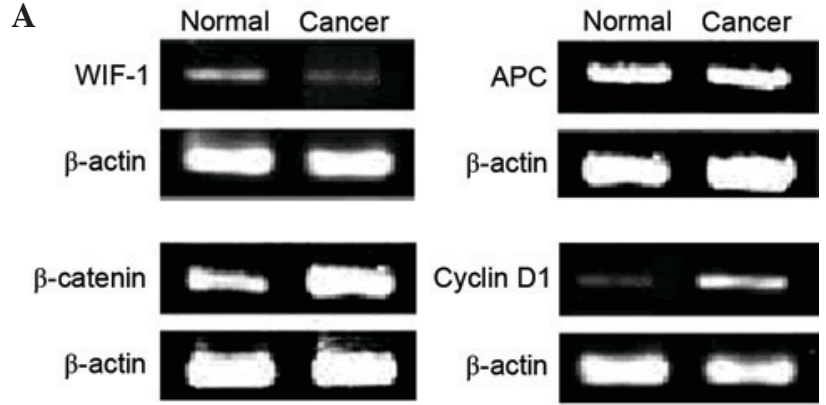

B

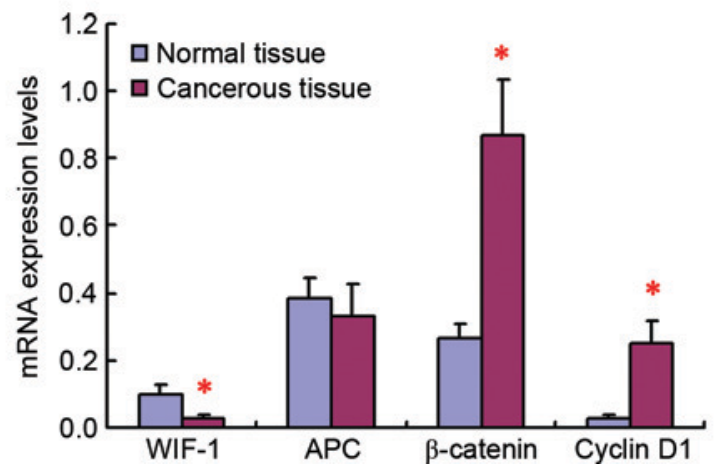

Figure 1. mRNA expression levels of WIF-1, APC, $\beta$-catenin and cyclin D1 in non-small cell lung cancer tissue samples and paracarcinoma tissue samples. The mRNA levels of WIF-1, APC, $\beta$-catenin and cyclin D1 were determined by reverse transcription-quantitative polymerase chain reaction, and $\beta$-actin served as the loading control. (A) Representative images of mRNA expression in cancerous tissues from adenocarcinoma or squamous carcinoma cases and in adjacent tissues to the carcinoma. (B) Analysis of the ratios of each target band density to that of $\beta$-actin in each case for four genes in normal tissues and cancerous tissues. Data are shown as means \pm standard deviation. " $\mathrm{P}<0.05$ compared with the normal group ( $\mathrm{n}=52)$. WIF-1, Wnt inhibitory factor-1; APC, adenomatous polyposis coli; mRNA, messenger RNA.

$(\mathrm{P}=0.930, \mathrm{P}=0.420, \mathrm{P}=0.638$ and $\mathrm{P}=0.438$, respectively). The clinicopathological characteristics and statistical analysis of these cases are displayed in Table II.

mRNA expression levels of WIF-1,APC, $\beta$-catenin and cyclin D1. The RT-qPCR results revealed that WIF-1, APC, $\beta$-catenin and cyclin D1 mRNA expression was present both in cancer tissue samples and in paracarcinoma tissue samples (Fig. 1). No obvious difference could be observed for APC mRNA expression levels between lung cancer tissues and adjacent tissues to the carcinoma. However, compared with normal tissues, the mRNA expression level of WIF-1 was significantly downregulated, while the $\beta$-catenin and cyclin D1 mRNA expression levels were remarkably increased, in tumor tissues. Importantly, the mRNA expression of cyclin D1 was remarkably low in normal lung tissues, but was markedly upregulated in tumor tissues.

Immunohistochemical staining detection of the protein expression levels of WIF-1, $\beta$-catenin, cyclin DI and $A P C$. As shown by immunohistochemical staining, WIF-1, $\beta$-catenin, cyclin D1 and APC exhibited positive expression both in cancer tissue samples and in paracarcinoma lung tissue samples (Fig. 2). The proteins levels of $\beta$-catenin and cyclin D1 were much higher in cancer tissues than in paracarcinoma tissues. $\beta$-catenin was mainly expressed in the cytoplasm. The upregulation rates of $\beta$-catenin and

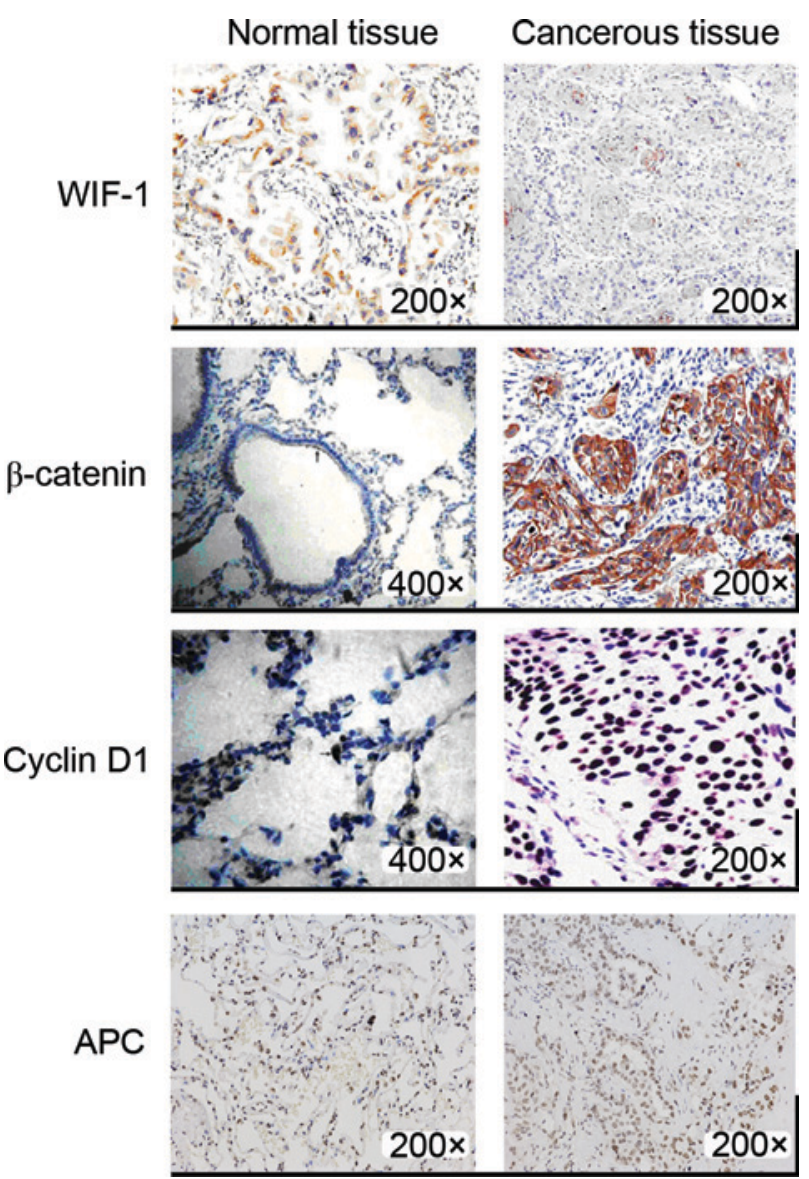

Figure 2. Differences in the protein expression levels of WIF-1, $\beta$-catenin, cyclin D1 and APC in non-small cell lung cancer tissue samples and paracarcinoma tissue samples. The protein levels of WIF-1, $\beta$-catenin, cyclin D1 and APC were detected by immunohistochemical staining. Representative images of cancerous tissues form adenocarcinoma or squamous carcinoma cases and normal tissues adjacent to the carcinoma are shown. WIF-1, Wnt inhibitory factor-1; APC, adenomatous polyposis coli.

cyclin D1 were 76.9 and $67.3 \%$ in lung tissue, respectively. WIF-1 expression was clearly decreased in cancer tissues compared with that in normal tissues, and its downregulation rate was $92.3 \%$. The same results were obtained for mRNA expression, and no obvious difference could be observed for APC protein expression levels between lung cancer tissues and adjacent tissues to the carcinoma. The results of the analysis of the association between the protein expression levels of WIF-1, $\beta$-catenin and cyclin D1 detected by immunohistochemical staining and the clinical characteristics of patients in the NSCLC group are shown in Table III. The percentage of patients with downregulated WIF-1 expression and positive lymph nodal metastasis is significantly higher than the percentage of patients who are negative for lymph nodal metastasis or exhibit upregulated WIF-1 expression with positive lymph nodal metastasis $(\mathrm{P}=0.006)$. The percentage of patients with upregulated cyclin D1 expression and positive lymph nodal metastasis is significant higher than that of patients with negative lymph nodal metastasis or those who exhibit downregulated cyclin D1 expression with positive lymph nodal metastasis $(\mathrm{P}=0.043)$. Furthermore, the percentage of patients with upregulated cyclin D1 expression and middle or low pathological classification was significantly 
Table III. Analysis of the association between the protein expression ${ }^{\mathrm{a}}$ of WIF-1, $\beta$-catenin and cyclin D1 and the clinical characteristics of patients with non-small cell lung cancer.

\begin{tabular}{|c|c|c|c|c|c|c|c|}
\hline \multirow[b]{2}{*}{ Characteristics } & \multirow[b]{2}{*}{$\mathrm{N}$} & \multicolumn{2}{|c|}{ WIF-1 expression } & \multicolumn{2}{|c|}{$\beta$-catenin expression } & \multicolumn{2}{|c|}{ Cyclin D1 expression } \\
\hline & &,$+ \mathrm{n}(\%)$ &,$- \mathrm{n}(\%)$ &,$+ \mathrm{n}(\%)$ &,$- \mathrm{n}(\%)$ &,$+ \mathrm{n}(\%)$ &,$- \mathrm{n}(\%)$ \\
\hline \multicolumn{8}{|l|}{ Histopathology } \\
\hline Adenocarcinoma & 29 & $7(24.14)$ & $22(75.86)$ & $23(79.31)$ & $6(20.69)$ & $20(68.97)$ & $9(31.03)$ \\
\hline Squamous carcinoma & 23 & $5(7.70)$ & $18(92.30)$ & $17(73.91)$ & $6(26.09)$ & $15(65.22)$ & $8(34.78)$ \\
\hline \multicolumn{8}{|l|}{ Lymph nodal metastasis } \\
\hline Positive & 39 & $3(7.69)$ & $36(92.31)^{\mathrm{b}}$ & $31(79.49)$ & $8(20.51)$ & $29(74.36)^{\mathrm{b}}$ & $10(25.64)$ \\
\hline Negative & 13 & $6(46.15)$ & $7(53.85)$ & $6(46.15)$ & $7(53.85)$ & $5(38.46)$ & $8(61.54)$ \\
\hline \multicolumn{8}{|c|}{ Pathological classification } \\
\hline High & 20 & $8(40.00)$ & $12(60.00)$ & $12(60.00)$ & $8(40.00)$ & $11(55.00)$ & $9(45.00)$ \\
\hline Middle/low & 32 & $6(18.75)$ & $26(81.25)$ & $25(78.13)$ & $7(21.87)$ & $28(87.50)^{\mathrm{a}}$ & $4(12.50)$ \\
\hline \multicolumn{8}{|l|}{ Clinical stage } \\
\hline I & 24 & $7(29.17)$ & $17(70.83)$ & $14(58.33)$ & $10(41.67)$ & $12(50.00)$ & $12(50.00)$ \\
\hline II/III & 28 & $4(14.29)$ & $24(85.71)$ & $21(75.00)$ & $7(25.00)$ & $18(64.29)$ & $10(35.71)$ \\
\hline
\end{tabular}

${ }^{a}$ The integrated optical density values of each target protein in each case were analyzed in normal and cancerous tissues. ' + ' was assigned when the expression level in lung tissues was higher than that in paracarcinoma tissues; '-' was assigned when the expression level in lung tissues was equal or lower than that in paracarcinoma tissues. The $\chi^{2}$ test was used to analyze the differences in the values of each variable. ${ }^{b} \mathrm{P}<0.05$. WIF-1, Wnt inhibitory factor-1.

higher than the percentage of patients with high pathological classification or with downregulated cyclin D1 expression and middle/low pathological classification $(\mathrm{P}=0.008)$.

\section{Discussion}

During the tumorigenesis of lung cancer, more attention should be paid to the regulatory effect of WIF-1, which is the upstream factor in the Wnt signaling pathway (9), as well as to the activation and regulation of Wnt signal transduction under different physiological and pathological conditions. Exploring the role of Wnt signaling pathways in the activating process could provide a theoretical basis and novel targets for the clinical diagnosis and treatment of lung cancer.

It has been confirmed that the abnormality of the Wnt signaling pathway is closely associated with malignant tumors, including colorectal cancer, melanoma, NSCLC, head and neck cancer, leukemia and mesothelioma (14). Mainly absence or downregulation of WIF-1 expression could be observed in these tumors, which correlates with signal transduction (15). It was reported that, compared with normal tissues, decreased WIF-1 expression could happen in $80 \%$ of esophageal cancer cases, $75 \%$ of gastric cancer cases, $74.6 \%$ of colon cancer cases and $66 \%$ of pancreatic cancer cases (15). However, there was no obvious correlation between reduced WIF-1 expression and the clinicopathological characteristics of the tumor (15). In NSCLC, the downregulated or missing expression of WIF-1 could be detected in $\sim 72 \%$ of cases.

In the present study, the expression of various important factors in the Wnt signaling pathway was detected, including $\beta$-catenin, APC and cyclin D1. Furthermore, the negative regulatory factors of WIF-1 expression were also detected. The results indicated that, compared with normal tissues, a marked decrease in WIF-1 expression and an increase in $\beta$-catenin and cyclin D1 expression could be observed in tumor tissues, indicating Wnt signaling activation. In lung cancer tissues, $\beta$-catenin protein with position transfer is mainly expressed in cytoplasm other than in cytomembrane as normal lung tissue. The activation of the Wnt signaling pathway may be associated with human lung cancer (16). Repressing WIF-1 expression could act as an initial promoter in the activation of Wnt signal transduction. Since the expression of APC was not altered in the present study, it is possible to conclude that the Wnt signaling pathways activated in NSCLC may differ from those in other tumors.

With the in-depth study of Wnt signaling pathways, WIF-1 as an inhibitory factor and its association with the signaling pathway is attracting more and more attention. WIF-1 inhibits the activity of Wnt signaling pathways by directly binding to Wnt proteins (17). In normal tissues of the human body, with the exception of the retina (which is the tissue where WIF-1 was initially identified), the presence of WIF-1 was detected in a variety of organs such as the lung, prostate, brain and skeletal muscle (17).

At present, the interaction mechanism of WIF-1 and Wnt is still not fully clear. A previous study reported that WIF-1 displayed different inhibition mechanisms with different Wnt molecules (18). There are at least two types of Wnt proteins that could interact with human WIF-1 to form a non-covalent complex with highly specific affinity in vitro (18). WIF-1 and Wnt4 are both components of the extracellular matrix (18). It was reported that WIF-1 could combine and form complex with Wnt4, thereby inhibiting Wnt4 to transduce any intracellular signal. Wnt signaling pathways could be inhibited due to the action of WIF-1, which consequently inhibits cell growth and differentiation (19). By reducing WIF-1 expression, Wnt signaling can be activated, which promotes cell growth and proliferation (19-21). 
The structure of WIF-1 is known. Although it has been confirmed that WIF-1 can be used as a negative feedback regulatory factor for Wnt signaling pathways (22), its own regulation and the interaction mechanism between Wnt signaling pathways and WIF-1 expression regulation are still not fully clear. Previous studies have confirmed that certain reactive elements of T-cell factor (TCF) cells exist in the gene promoter region of WIF-1, and that TCF cells are important nuclear target factors for Wnt signaling pathways (23). The activation of Wnt signaling could induce $\beta$-catenin accumulation in the cytoplasm and nuclei entrance, thereby enabling the identification of transcription factors such as lymphatic enhancement factor, $\mathrm{T}$ cell-related factors and activating target genes, which ultimately control embryonic development, cell growth, differentiation and apoptosis $(24,25)$. Notably, the time and spatial distribution of extracellular WIF-1 may be associated with its different affinity towards different Wnt molecules (26). Therefore, Wnt signaling may serve a role in the regulation of the extracellular space distribution of WIF-1, although this must be confirmed by further studies. Hypermethylation of the promoter region of the gene coding for WIF-1 could lead to the post-transcriptional silencing of the WIF-1 gene, and thereby could induce absent or downregulated WIF-1 expression, eventually causing abnormal activation of Wnt signaling pathways and the corresponding changes (26). Whether hypermethylation of the WIF-1 promoter can be used as an effective detection marker for related diseases such as cancer and osteoarthrosis, and whether demethylation of the WIF-1 gene to restore its expression could be a potential target for the treatment of these diseases, requires future investigation.

The present study demonstrated that WIF-1, which is the upstream gene in the $\mathrm{Wnt} / \beta$-catenin signaling pathway, acts as the reverse suppressor of Wnt, and its expression is significantly decreased in lung cancer tissues. In addition, the expression levels of $\beta$-catenin and cyclin D1, which are an important transcription factor and the downstream target gene of Wnt, respectively, were increased in lung cancer tissues. These changes indicate that Wnt signaling pathways can be activated in NSCLC, and may be closely associated with lymph nodal metastasis and lower pathological classification.

In summary, the present study demonstrated that the activation of the Wnt/ $\beta$-catenin signaling pathway in lung cancer tissues is initiated by WIF-1 gene inhibition. Since no differences in APC expression in NSCLC and non-cancerous tissues were observed, the activation of this signaling pathway may be different between NSCLC and other tumors.

\section{Acknowledgements}

The present study was supported by the Science and Technology Support Project of Xinjiang Uygur Autonomous Region (grant no. 2016E02071) and the Tianjin Health and Family Planning Commission of Science and Technology Project Foundation (grant no. 16KG153).

\section{References}

1. Stewart DJ: Wnt signaling pathway in non-small cell lung cancer. J Natl Cancer Inst 106: djt356, 2014.

2. Chen X, Meng J, Yue W, Yu J, Yang J, Yao Z and Zhang L: Fibulin-3 suppresses Wnt/ $\beta$-catenin signaling and lung cancer invasion. Carcinogenesis 35: 1707-1716, 2014.
3. Pacheco-PinedoEC and Morrisey EE: Wnt and Kras signaling-dark siblings in lung cancer. Oncotarget 2: 569-574, 2011.

4. Nelson WJ and Nusse R: Convergence of Wnt, beta-catenin, and cadherin pathways. Science 303: 1483-1487, 2004.

5. Wend P, Holland JD, Ziebold U and Birchmeier W: Wnt signaling in stem and cancer stem cells. Semin Cell Dev Biol 21: 855-863, 2010.

6. Rubin EM, Guo Y, Tu K, Xie J, Zi X and Hoang BH: Wnt inhibitory factor 1 decreases tumorigenesis and metastasis in osteosarcoma. Mol Cancer Ther 9: 731-741, 2010.

7. Esteller M: Cancer epigenomics: DNA methylomes and histone-modification maps. Nat Rev Genet 8: 286-298, 2007.

8. Liu YL, Yang HP, Gong L, Tang CL and Wang HJ: Hypomethylation effects of curcumin, demethoxycurcumin and bisdemethoxycurcumin on WIF-1 promoter in non-small cell lung cancer cell lines. Mol Med Rep 4: 675-679, 2011.

9. Gao Z, Xu Z, Hung MS, Lin YC, Wang T, Gong M, Zhi X, Jablons DM and You L: Procaine and procainamide inhibit the Wnt canonical pathway by promoter demethylation of WIF-1 in lung cancer cells. Oncol Rep 22: 1479-1484, 2009.

10. Travis WD, Brambilla E, Nicholson AG, et al; WHO Panel: The 2015 World Health Organization classification of lung tumors: Impact of genetic, clinical and radiologic advances since the 2004 classification. J Thorac Oncol 10: 1243-1260, 2015.

11. Warth A,Muley T,Meister M,Stenzinger A, Thomas M,Schirmacher P, Schnabel PA, Budczies J, Hoffmann H and Weichert W: The novel histologic International Association for the Study of Lung Cancer/American Thoracic Society/European Respiratory Society classification system of lung adenocarcinoma is a stage-independent predictor of survival. J Clin Oncol 30: 1438-1446, 2012.

12. Li Q, Hu X, Bai Y, Alattar M, Ma D, Cao Y, Hao Y, Wang L and Jiang $\mathrm{C}$ : The oxidative damage and inflammatory response induced by lead sulfide nanoparticles in rat lung. Food Chem Toxicol 60: 213-217, 2013.

13. Zhang S, Jiang C, Liu H, Guan Z, Zeng Q, Zhang C, Lei R, Xia T, Gao H, Yang L, et al: Fluoride-elicited developmental testicular toxicity in rats: Roles of endoplasmic reticulum stress and inflammatory response. Toxicol Appl Pharmacol 271: 206-215, 2013.

14. Le PN, McDermott JD and Jimeno A: Targeting the Wnt pathway in human cancers: Therapeutic targeting with a focus on OMP-54F28. Pharmacol Ther 146: 1-11, 2015.

15. Surmann-Schmitt C, Sasaki T, Hattori T, Eitzinger N, Schett G, von der Mark K and Stock M: The Wnt antagonist Wif-1 interacts with CTGF and inhibits CTGF activity. J Cell Physiol 227: 2207-2216, 2012.

16. Li ZL, Shao SH, Jiao F, Yue Z and Ma Y: Cyclin D1 regulates lung cancer invasion and metastasis. Sheng Li Xue Bao 64: 55-61, 2012 (In Chinese).

17. Xu X, Sun PL, Li JZ, Jheon S, Lee CT and Chung JH: Aberrant Wnt $1 / \beta$-catenin expression is an independent poor prognostic marker of non-small cell lung cancer after surgery. $J$ Thorac Oncol 6: 716-724, 2011.

18. Hunter DD, Zhang M, Ferguson JW, Koch M and Brunken WJ: The extracellular matrix component WIF-1 is expressed during, and can modulate, retinal development. Mol Cell Neurosci 27: 477-488, 2004.

19. Lin YC, You L, Xu Z, et al: Wnt inhibitory factor-1 gene transfer inhibits melanoma cell growth. Hum Gene Ther 18: 379-386, 2007.

20. Alamgeer M, Peacock CD, Matsui W, Ganju V and Watkins DN: Cancer stem cells in lung cancer: Evidence and controversies. Respirology 18: 757-764, 2013.

21. Fang Y, Wang L, Zhang Y, Ge C and Xu C: Wif-1 methylation and $\beta$-catenin expression in colorectal serrated lesions. Zhonghua Bing Li Xue Za Zhi 43: 15-19, 2014 (In Chinese).

22. Yang TM, Leu SW, Li JM, et al: WIF-1 promoter region hypermethylation as an adjuvant diagnostic marker for non-small cell lung cancer-related malignant pleural effusions. J Cancer Res Clin Oncol 135: 919-924, 2009.

23. Cadigan KM and Waterman ML: TCF/LEFs and Wnt signaling in the nucleus. Cold Spring Harb Perspect Biol 4: pii: a007906, 2012.

24. Beyer C, Schramm A, Akhmetshina A, et al: $\beta$-catenin is a central mediator of pro-fibrotic Wnt signaling in systemic sclerosis. Ann Rheum Dis 71: 761-767, 2012.

25. Roberts DM, Pronobis MI, Poulton JS, Kane EG and Peifer M: Regulation of Wnt signaling by the tumor suppressor adenomatous polyposis coli does not require the ability to enter the nucleus or a particular cytoplasmic localization. Mol Biol Cell 23: 2041-2056, 2012.

26. Bányai L, Kerekes K and Patthy L: Characterization of a Wnt-binding site of the WIF-domain of Wnt inhibitory factor-1. FEBS Lett 586: 3122-3126, 2012. 